\title{
Article \\ Sundew-Inspired Adhesive Hydrogel Threads through Reversible Complexation of Polyphenol and Boronic Acid
}

\author{
Jae Hyuk Choi ${ }^{1}$, Donghee Son ${ }^{2,3,4, * \mathbb{D}}$ and Mikyung Shin $1,3,5, * \mathbb{D}$ \\ 1 Department of Intelligent Precision Healthcare Convergence, Sungkyunkwan University (SKKU), \\ Seobu-ro 2066, Jangan-gu, Suwon 16419, Korea; wre56@naver.com \\ 2 Department of Electrical and Computer Engineering, Sungkyunkwan University (SKKU), \\ Seobu-ro 2066, Jangan-gu, Suwon 16419, Korea \\ 3 Center for Neuroscience Imaging Research, Institute for Basic Science (IBS), Suwon 16419, Korea \\ 4 Department of Superintelligence Engineering, Sungkyunkwan University, Suwon 16419, Korea \\ 5 Department of Biomedical Engineering, Sungkyunkwan University (SKKU), Suwon 16419, Korea \\ * Correspondence: daniel3600@g.skku.edu (D.S.); mikyungshin@g.skku.edu (M.S.)
}

Citation: Choi, J.H.; Son, D.; Shin, M. Sundew-Inspired Adhesive Hydrogel Threads through Reversible Complexation of Polyphenol and Boronic Acid. Appl. Sci. 2021, 11, 8591. https://doi.org/10.3390/ app11188591

Academic Editor: Agnese Magnani

Received: 22 August 2021

Accepted: 14 September 2021

Published: 16 September 2021

Publisher's Note: MDPI stays neutral with regard to jurisdictional claims in published maps and institutional affiliations.

Copyright: (c) 2021 by the authors. Licensee MDPI, Basel, Switzerland. This article is an open access article distributed under the terms and conditions of the Creative Commons Attribution (CC BY) license (https:// creativecommons.org/licenses/by/ $4.0 /)$.

\begin{abstract}
Adhesive hydrogels have been utilized as tissue sealants, hemostatic agents, and wound dressings, with the aim of replacing conventional sutures. To prevent immune response and serious inflammation from those hydrogels after sealing, natural biocompatible polysaccharides are widely used as a component of the hydrogels. However, the weak mechanical strength, insufficient adhesiveness, and rapid dissociation of the hydrogels necessitates additional suturing at the wound site. In this study, we report on a solid polysaccharide thread reversibly crosslinked with boronic acidpolyphenol complexation and its adhesive gelation for complete tissue sealing without additional suturing. The polysaccharide adhesive suture threads demonstrated long-term stability, which is useful when used for skin wound suturing. Specifically, their robust adhesion property occurred as soon as the threads were soaked, attaining a swollen hydrogel state, dependent on the presence of body fluids after suturing. Moreover, the $\mathrm{pH}$ of the body fluids affects the viscoelasticity and adhesiveness of the hydrogels in order to ensure a tight sealing. Therefore, we expect that these $\mathrm{pH}$-responsive adhesive threads would be promising for the development of on-demand functional suture materials.
\end{abstract}

Keywords: bioadhesive; hydrogel threads; $\mathrm{pH}$ responsiveness

\section{Introduction}

Suturing is the traditional method used to seal biological tissues during surgical interventions. Although many medical glues [1] have been developed to replace suturing, conventional suturing is still dominantly adopted in preference to glues in medical centers due to ease of use and secure tissue sealing without mechanical delamination after surgery. Such suture threads, composed of biodegradable polymers (e.g., poly (lactic-co-glycolic acid)), may potentially result in acute/chronic inflammation by evoking continuous frictional stress on the tissues, and due to their mechanical stiffness and to acidic byproducts after degradation [2]. Furthermore, suturing suffers from the critical challenges associated with undesired issues such as the application of excessive pressure on the site of election, low biocompatibility, and difficult biodegradation time control [3-5]. Thus, to replace this conventional sealing method, a new type of adhesive material with thread formulation would be suitable.

As an alternative to existing sutures, various studies have been focused on developing bioadhesives, with improved biocompatibility [6,7], controllable degradation [8,9], and drug encapsulation [10,11]. However, these adhesives still have weak mechanical properties and low adhesiveness when compared to those of conventional suturing. To overcome these issues, many researchers have been interested in fabricating bio-adhesive 
fibers with strong mechanical strength [12]. For instance, one of the studies showed that a wet-spinning technique applied to alginate/chitosan fibers achieved the mechanical properties of a Young's modulus 200\% higher than that of the natural materials [13]. Another study showed the formation of tough hydrogels, rehydrated from organogels, allowing further spinning to fibrous structures [14]. Furthermore, a mechanically robust hydrogel fiber fabricated by using a dynamic host-guest crosslinking that mimics the silk fibers of a spider web [15], and a viscoelastic hydrogel with a nanofiber structure similar to the sundew adhesive have been developed [16]. Nevertheless, these hydrogels cannot be adopted for versatile tissues with different physiological $\mathrm{pHs}$, for instance, the strong acidity of the stomach and the mild basicity of the intestines.

In this study, we report a $\mathrm{pH}$-responsive adhesive hydrogel thread that mimics sundew adhesive mucus (Figure 1a). By simply mixing boronic acid-conjugated alginate (AlBA) and tannic acid (TA), which is an abundant polyphenol found in plants, viscoelastic gelation of the adhesive hydrogel thread occurs via reversible boronic acid-cis-diol complexation (Figure 1b, left) [17], further allowing facile spinning to fabricate hydrogel fibers (Figure 1b, right). After complete dehydration of the hydrogel fiber, the thread with strong mechanical strength is prepared for use in suturing like conventional threads. The thickness of the thread can be precisely controlled through a layer-by-layer stacking process of the hydrogel fibers. In particular, after suturing, the adhesive threads on the tissues can be swollen under exposure to a physiological buffer ( $\mathrm{pH}$ 7.4), and their mechanical modulus is higher at acidic $\mathrm{pH}$ than that at neutral $\mathrm{pH}$, owing to a pronounced precipitation tendency of the alginate as alginic acid in acidic conditions [18].

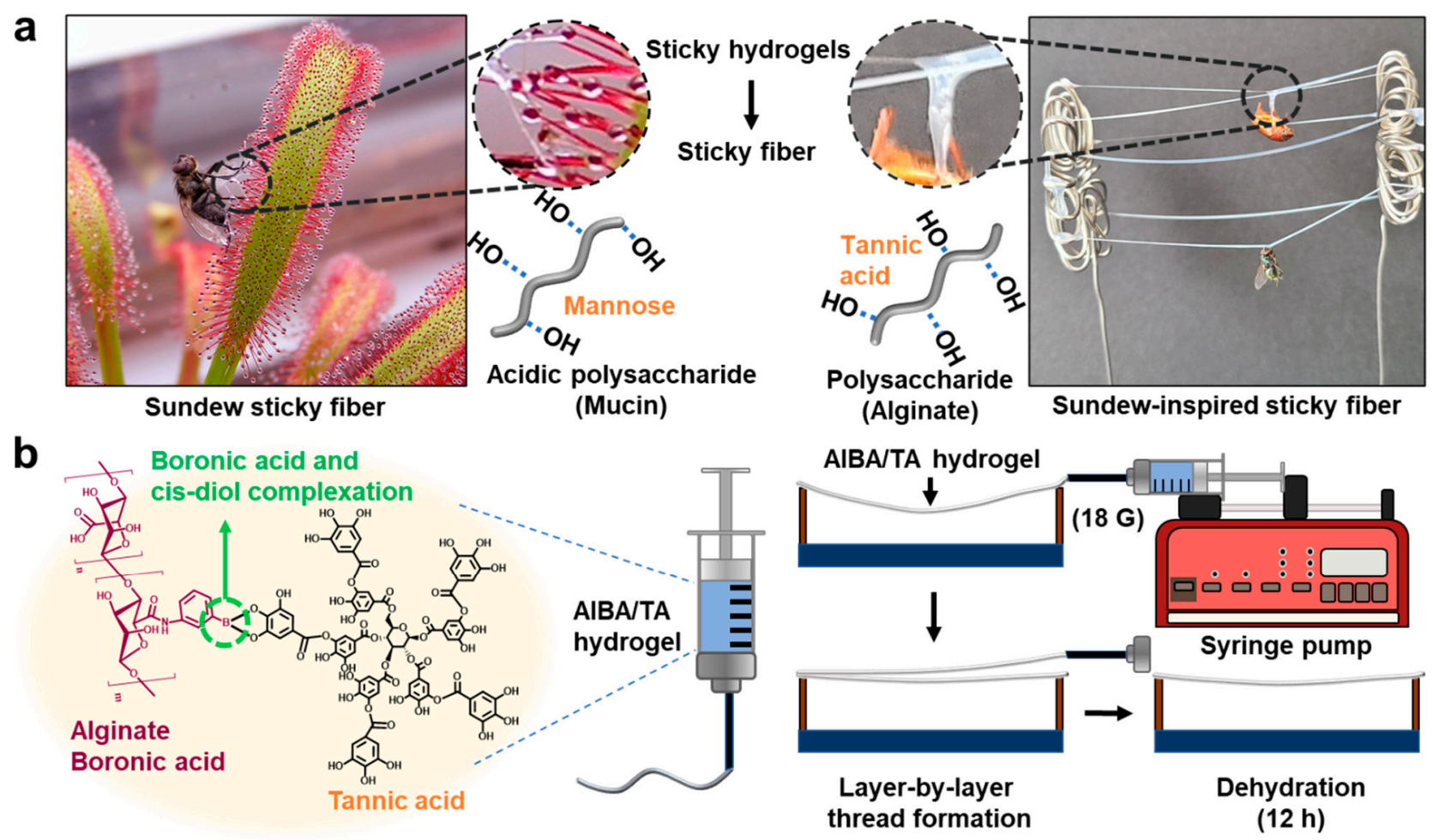

Figure 1. (a) Schematic of a hydrogel formed from sundew and an AlBA/TA hydrogel inspired by it. It is composed of mono-/polysaccharides, which are the main components of the sticky hydrogel, and a crosslinking agent that helps the shape to be thread-like. (b) The chemical structure of the complexation between boronic acid and cis-diol in TA (left) and overall scheme for the manufacturing process of the AlBA/TA hydrogel threads (right). 


\section{Materials and Methods}

2.1. Materials

Alginic acid sodium salt from brown algae (medium viscosity), 3-Aminophenyl boronic acid (BA), N-hydroxysuccinimide (NHS), HEPES buffer solution, trypan blue, BisTris base, sodium acetate, pyrogallol, pyrocatechol, and tannic acid (TA) were purchased from Sigma Aldrich (St. Louis, MO, USA), 1-(3-dimethylaminopropyl)-3-ethylcarbodiimide hydrochloride (EDC-HCl) was purchased from Tokyo Chemical Industry (Tokyo, Japan), phosphate-buffered saline (PBS, 10×, pH 7.2) was purchased from WELGENE (Gyeongsan, Korea). SpectraPor 1 Dialysis Membrane (Standard RC Tubing, molecular weight cut off $(\mathrm{MWCO})=6-8 \mathrm{kDa}$ ) was purchased from Spectrum (Rancho Dominguez, CA, USA) and used as received.

\subsection{Synthesis and Characterization of $A l B A$}

For AlBA synthesis, a two-step condensation reaction between alginate (involving $\mathrm{COOH}$ groups) and BA ( $\mathrm{NH}_{2}$ groups) via EDC/NHS coupling agents was carried out following an algorithm published elsewhere [19]. Briefly, $1 \mathrm{~g}$ of alginate was dissolved in $250 \mathrm{~mL}$ of MES buffer (0.1 M, pH 5.0). Subsequently, EDC-HCl (700 mg) and NHS (100 mg) dissolved in $10 \mathrm{~mL}$ of deionized water (DDW) were added dropwise to the solution for $10 \mathrm{~min}$. Thereafter, boronic acid (BA, $300 \mathrm{mg}$ ) dissolved in $10 \mathrm{~mL}$ of DDW was added dropwise to the alginate/EDC/NHS solution and left overnight. After the reaction was completed, the solution was dialyzed in DDW for 3 days, during which the DDW (5 L with sodium chloride $45 \mathrm{~g}$ ) was changed every $4 \mathrm{~h}$. Finally, the solution was dialyzed against pure DDW for $4 \mathrm{~h}$. The dialyzed solution was freeze-dried for four days and stored in a refrigerator. In the synthesized AlBA, the degree of substitution of boronic acid in alginate was $39.2 \% \pm 1.2 \%$, and the absorbance at $243 \mathrm{~nm}\left(\mathrm{~A}_{243}\right)$ was measured by an Agilent 8453 UV-vis spectrometer (Agilent Technologies, Santa Clara, CA, USA). The 3-aminophenyl boronic acid solutions (ranging from $6.25-30 \mu \mathrm{g} / \mathrm{mL}$ ) were used to obtain a calibration curve.

\subsection{AlBA/TA Hydrogel Thread Formation}

To prepare the AlBA/TA hydrogel threads, an amount of AlBA was dissolved in DDW to a final concentration of $3 \mathrm{wt} \%$, and TA was mixed into the solution to a final concentration of $0.01 \mathrm{M}$. The prepared hydrogel was centrifuged at $2000 \mathrm{rpm}$ for $20 \mathrm{~min}$ to remove the residual air bubbles by loading it into a $1 \mathrm{~mL}$ syringe. Subsequently, the solution was injected at a rate of $20 \mu \mathrm{L} / \mathrm{min}$ from an 18-gauge blunt needle, and the hydrogel was drawn at a rate of $25 \mathrm{~mm} / \mathrm{min}$. The hydrogel released from the syringe was dried for $12 \mathrm{~h}$. Two-layer and three-layer threads were prepared by stacking in the hydrogel state before the drying phase.

\subsection{Rheological Characterization}

Rheological properties (storage (elastic) modulus $\mathrm{G}^{\prime}$ and loss (viscous) modulus $\mathrm{G}^{\prime \prime}$ ) of $\operatorname{AlBA}(1,2,3$, and $4 \mathrm{wt} \%)$ with TA and phenol derivatives $(0.01 \mathrm{M})$ were analyzed using a Discovery Hybrid Rheometer 2 (TA Instrument, New Castle, DE, USA) with a 20-mm parallel-plate geometry. The frequency sweeps ranged from 0.1 to $10 \mathrm{~Hz}$ at $1 \%$ strain.

\subsection{Measurement of Mechanical Properties}

The diameters of the threads were measured using a Leica DMi 8 inverted microscope with Leica LAS X software and a scanning electron microscope (SEM; JSM-6309A, JEOL USA, Inc., Peabody, MA, USA). To analyze the maximum load (N) of the threads and their suturing strength on the skin tissue, a universal testing machine (UTM; Instron 5943, Norfolk, MA, USA and QC-508E, Cometech Co., Ltd., Taichung, Taiwan) was used. The threads that dried completely at room temperature were cut into pieces of $3 \mathrm{~cm}$ length to be used as samples. To analyze the effect of $\mathrm{pH}$ on the threads, the samples were swollen in universal buffer (consisting of $20 \mathrm{mM}$ HEPES, $20 \mathrm{mM}$ Bis-Tris, and $20 \mathrm{mM}$ sodium acetate) 
solution for $1 \mathrm{~h}$. For the ex vivo test, the back skin of an 8-week-old rat and a 2-layer thread (with trypan blue $v / v$ 10\% for dyeing) were used. Shear stress was applied using a $100 \mathrm{~N}$ load cell at a constant rate of $20 \mathrm{~mm} / \mathrm{min}$.

\subsection{Release of TA from the AlBA/TA Hydrogel Thread}

A 24-well transwell $\left(8.0 \mu \mathrm{m}\right.$ polycarbonate membrane, Costar ${ }^{\circledR}$ (Washington, DC, USA) was used to measure the extent of tannic acid release from the two-layer thread. The $1 \mathrm{~cm}$ thread pieces were placed in a transwell containing $0.2 \mathrm{~mL}$ of PBS $(1 \times)$, then placed in a 24-well plate containing $0.8 \mathrm{~mL}$ of PBS $(1 \times)$ and replaced with an equivalent volume of fresh PBS after each aliquot had been used for analysis. The measurement was conducted nine times per day for 1 day to observe the initial release ability, and then at intervals of 1-6 days. Tannic acid emission concentrations were measured spectrophotometrically (UV-vis) by considering the absorbance at $279 \mathrm{~nm}$, directly correlated with the calibration curve obtained for the TA concentrations range of 5-20 $\mu \mathrm{g} / \mathrm{mL}$.

\subsection{Statistical Analysis}

Statistical significance was evaluated using a one-way ANOVA test with a post hoc Tukey test.

\section{Results and Discussion}

\subsection{Characterization of $A l B A$}

The AlBA polymers used to form the thread-like hydrogel can mutually and reversibly react owing to the simultaneous presence of "cis-diol" and "boronic acid" moieties on the single alginate backbone to have enhanced viscoelastic properties of the intrinsically crosslinked system [19]. The presence of the boronic acid moiety anchored onto the alginate chain was assessed spectrophotometrically by measuring the absorbance at $243 \mathrm{~nm}$ (Figure 2a). The degree of substitution (DOS) was calculated to be $39.2 \% \pm 1.2 \%$.

\subsection{Rheological Behavior of AlBA/TA Hydrogel Thread}

In attempting to obtain an AlBA hydrogel, the AlBA concentration was increased to $3 \mathrm{wt} \%$ to avoid the formation of persistent bubbles. As a result, the system became much more viscous instead of gelation as expected. However, to transform the concentrated solution of AlBA into a hydrogel, the classical route of alginate gelation with $\mathrm{CaCl}_{2}$ [20] was replaced by a new approach, in which polyphenol molecules were used as crosslinking agent for AlBA chains. Thus, AlBA was mixed with pyrogallol, pyrocatechol, or TA at a final polyphenol concentration of $0.01 \mathrm{M}$. In the case of the AlBA/TA mixture, the gelation occurs immediately after TA addition, while for AlBA/phenol derivative systems a decrease in the overall viscosity was noticed. As shown in Figure $2 b$, as a result of rheological measurements, the values of loss modulus of the AlBA $3 \mathrm{wt} \%$ solution were higher than those of storage modulus over the entire range of the operating frequency $(0.1-10 \mathrm{~Hz})$, indicating a prevalent viscous component of the viscoelastic behavior. Similarly, but at generally smaller values, the system's AlBA/phenol derivatives roughly exhibited the same shift between moduli, showing a prevailing viscous component of the viscoelastic behavior. As a result, mixing AlBA with compounds carrying a single "cis-diol" moiety per molecule did not lead to intermolecular aggregation/crosslinking to make the initial solution a hydrogel. TA, having at least two "active cis-diol" functionalities per molecule, played the effective role of crosslinker between AlBA polymers, with direct consequences on the gel-like character of the final system revealed by the higher values of storage modulus in comparison with those of loss modulus (Figure $2 \mathrm{~b}$ ). To optimize the process of hydrogel formation by using TA as crosslinker, a number of AlBA/TA mixtures were prepared by varying AlBA concentration $(1,2,3$ and $4 \mathrm{wt} \%)$ at the same TA content $(0.01 \mathrm{M})$. In fact, at a concentration of $1 \mathrm{wt} \%$ AlBA, no gelation occurred, but at $2 \mathrm{wt} \% \mathrm{AlBA}$, the AlBA/TA mixture became a hydrogel. As the AlBA concentration increased, the number of polysaccharide-like molecules involved in crosslinking via TA also increased, so that at 
3 and $4 \mathrm{wt} \%$ AlBA, the hydrogels displayed relatively higher values of storage modulus (Figure 2c). However, it was noticed that the $4 \mathrm{wt} \%$ AlBA/0.01 M TA mixture exhibited a non-uniform appearance owing to persistent air bubbles inside the system during hydrogel formation and manipulation (Figure $2 \mathrm{~d}$ ). Taken together, $3 \mathrm{wt} \%$ of AlBA and $0.01 \mathrm{M}$ of TA were suitable for the facile fabrication of uniform hydrogel-based threads.
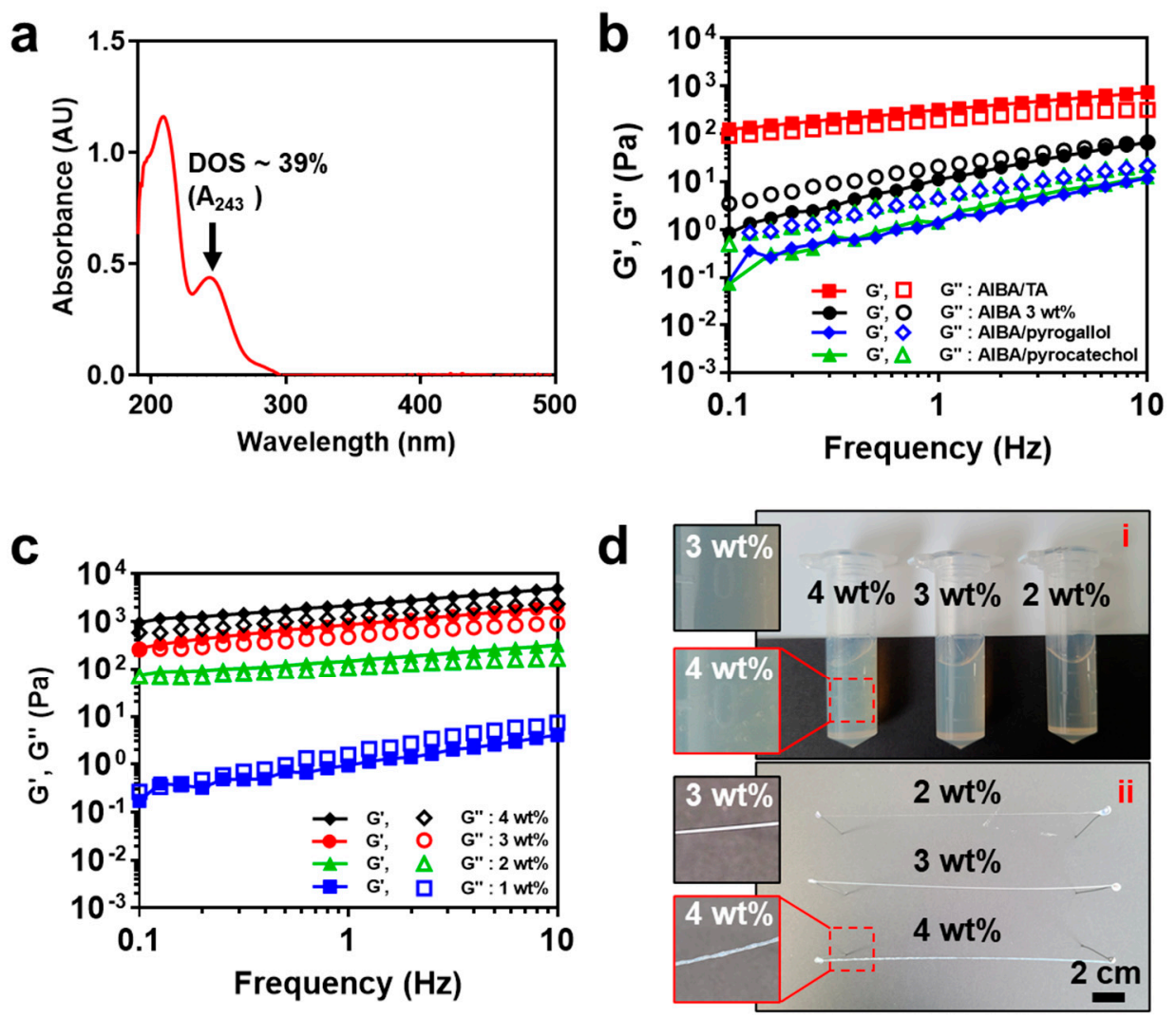

Figure 2. Physicochemical characteristics of AlBA/TA hydrogels. (a) UV-vis spectrum of AlBA aqueous solution. (b) Rheological properties of the mixtures of AlBA with various polyphenols: TA (red), pyrogallol (blue), pyrocatechol (green), and AlBA alone as a control (black). Storage modulus $\left(\mathrm{G}^{\prime}\right.$, filled symbols) and loss modulus $\left(\mathrm{G}^{\prime \prime}\right.$, open symbols). (c) Changes in rheological moduli of the AlBA/TA hydrogels as a function of AlBA concentration (1, 2, 3, and $4 \mathrm{wt} \%)$ at fixed molar concentration of TA $(0.01 \mathrm{M})$. (d) Photographs of AlBA solutions at different concentrations corresponding to the hydrogel fibers. Bubbles inside the solution (top red square) generate a rough surface hydrogel thread (bottom red square).

\subsection{Characterization of AlBA/TA Hydrogel Thread}

Most commercial suture threads are classified as "single" or "twisted". Most sutures use twisted threads to increase their mechanical strength. As there is no interaction between braiding, a complicated process is required, in which the threads must be woven multiple times to control the thickness. However, in the AlBA/TA hydrogel state, the thickness could be controlled more easily through the stacking process during layered thread formation, due to reversible crosslinking reactions, which mainly occurred between the boronic acid groups and the cis-diol moieties on the TA molecules. The sticky hydrogel spun through the syringe fused into a single final thread during the process of overlapping and drying. The superimposed hydrogels were not separated during the drying process. The thickness measurements of the manufactured threads were performed by optical microscopy. The thickness values of the one-layer, two-layer, and three-layer fabricated threads were $148.8 \pm 26.7 \mu \mathrm{m}, 217.7 \pm 35.2 \mu \mathrm{m}$, and $318.6 \pm 68.7 \mu \mathrm{m}$, respectively (Figure 3a). Supposing that an increased thickness might lead to undesirable voids and cracks inside the hydrogel, the strength of the fabricated threads was assessed by 
employing a universal tensile machine. The values of thread strength were $0.8 \pm 0.1 \mathrm{~N}$, $1.6 \pm 0.1 \mathrm{~N}$, and $2.5 \pm 0.4 \mathrm{~N}$ for one, two, and three layers, respectively (Figure 3b). SEM investigation confirmed that the thickness of the two-stacked layer threads is similar to that of a general suture $4.0(250 \mu \mathrm{m})$ (Figure 3c). At the same time, a linear dependence between strength and thickness of the threads was noticed. Therefore, this facilitates the ease of manufacture of threads with varying thicknesses.
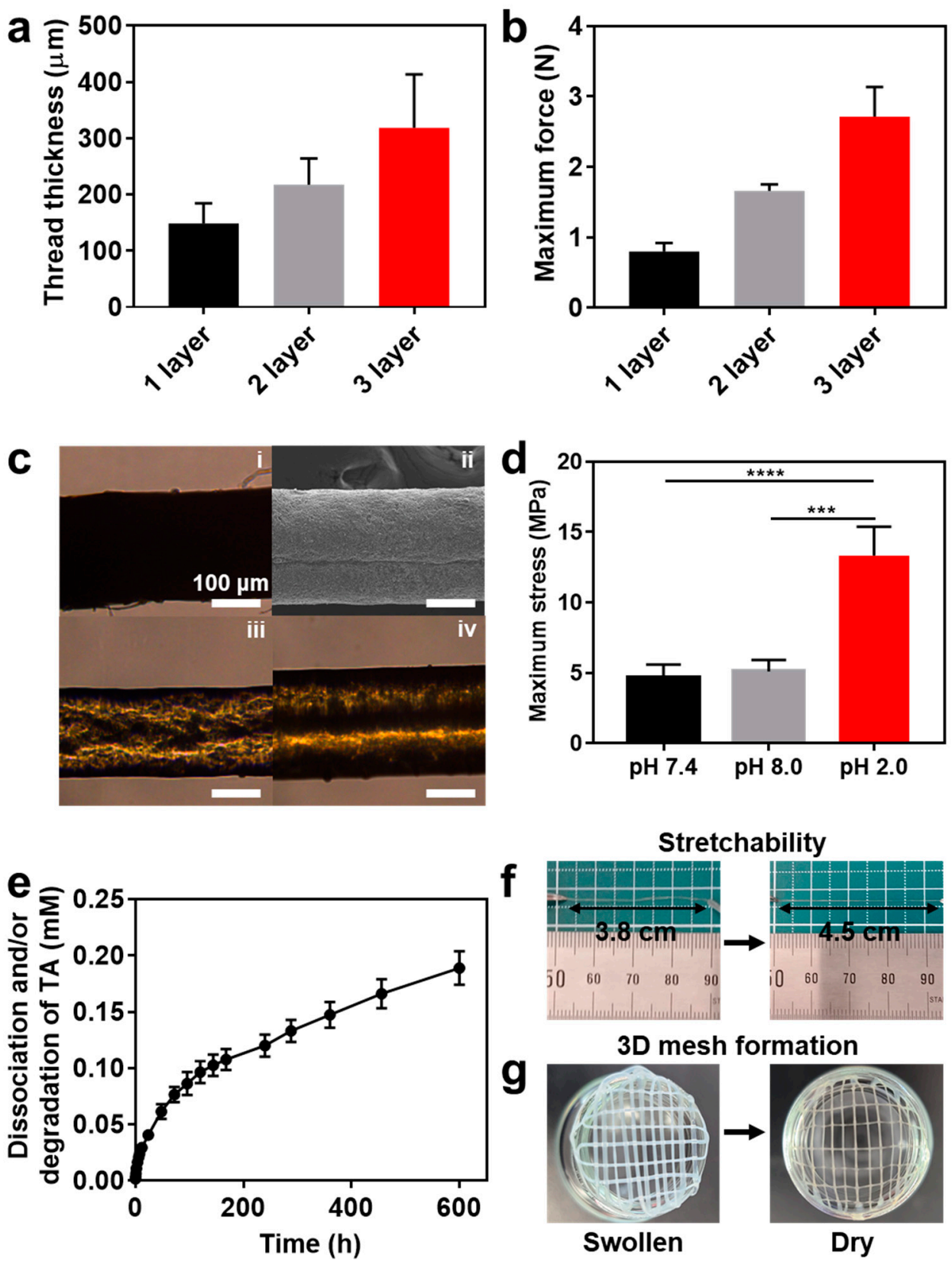

Figure 3. (a,b) Comparative observation of the thickness (a) and tensile maximum load (b) of adhesive AlBA/TA threads with different layers. (c) Optical microscopy images of commercial suture thread (i) and AlBA/TA threads (iii for 1 layer and iv for 2 layers). SEM image of AlBA/TA thread with 2 layers (ii). (d) Changes in maximum tensile stress of AlBA/TA threads with environment $\mathrm{pH}(n=3$ or more, mean $\pm \mathrm{SD})$ (one-way ANOVA, $\left.{ }^{* * *} p<0.001,{ }^{* * * *} p<0.0001\right)$. (e) Dissociation and/or degradation kinetics of TA over time from the AlBA/TA threads in a physiological buffer. (f) Stretchability of the swollen AlBA/TA threads at physiological condition. (g) 3D mesh constructs fabricated using the swollen AlBA/TA threads. 
AlBA, the main component of the thread, is $\mathrm{pH}$-sensitive [21]. Similar to alginate, which agglomerates at low $\mathrm{pH}$ [18], it was expected that alginate-like AlBA macromolecules would follow the same behavior at acidic $\mathrm{pH}$. Each organ in the body is characterized by a specific $\mathrm{pH}$ (stomach: 2.0, terminal ileum: 8.0) [22,23]. This may affect the thread performance. Therefore, we determined the change in the strength of the yarn (2 layer) based on $\mathrm{pH}$. The thread strength at $\mathrm{pH} 7.4$, known as the average physiological $\mathrm{pH}$ range in the human body, was $4.7 \pm 1.1 \mathrm{MPa}$; at $\mathrm{pH} 8.0$, the mean $\mathrm{pH}$ in the colon, the thread strength was $5.1 \pm 0.8 \mathrm{MPa}$; at $\mathrm{pH} 2.0$, the average $\mathrm{pH}$ in the stomach, the same quantity was $13.3 \pm 2.1 \mathrm{MPa}$ (Figure 3d). These results indicate that the AlBA/TA hydrogel thread could maintain sealing performance as a thread with higher tensile strength over a broad $\mathrm{pH}$ range.

TA is a natural polyphenolic compound derived from plants. The pyrogallol and catechol groups present in the molecular structure interact with AlBA to form a sticky hydrogel. Moreover, TA has excellent antioxidant, antibacterial, and hemostatic properties [24,25]. In addition, we observed that TA-bound AlBA/TA hydrogel threads continuously released tannic acids in the body. It was established that the release followed a linear tendency from the 4 th day to the 25th day after a burst release recorded during the first 3 days (total TA/its metabolites of $1.0 \mathrm{nmol}$ per hour) (Figure 3e). The result might be due to the dissociation and degradation of TA caused by hydrolysis of its ester groups. Interestingly, although the TA was degraded over time, the thread-like shape of the swollen hydrogel fiber was maintained for 25 days and its mechanical strength became slightly lower than that of the initial hydrogels (data not shown). According to previous reports, boronic acid can also have an intermolecular interaction with cis-diol in alginate backbone in $\mathrm{pH} 7.4[19,21]$. Thus, the partial complexation between boronic acid and alginate can help to maintain the fibrous shape even though the TA was continuously degraded from the hydrogels. Additionally, the hydrogel thread swollen in the physiological buffer can be stretched up to $118.4 \%$ of its original length (Figure 3f) and 3D mesh structures could be easily designed using these hydrogel fibers (Figure 3g).

\subsection{AlBA/TA Hydrogel Thread as a Tissue Suturing Application}

Sutures exert a pressure on the tissue at the site of application. This can lead to a reduced recovery and inflammation [26,27]. In contrast, hydrogels swell by means of body moisture and exhibit elasticity (Figure 4a). To verify this, AlBA/TA hydrogel threads were used to suture the skin of rats during testing to confirm whether the suture works efficiently and whether the skin was patched even after swelling. First, the dorsal skin of a rat was sutured seven times, after which PBS $(1 \times)$ was sprayed to swell the thread for $10 \mathrm{~min}$. Even after full swelling of the threads, no mechanical cracks in the swollen hydrogels were observed, and the tissue was well sealed (Figure $4 \mathrm{~b}$ ). In addition, to measure the fixing strength of the tissue, the maximum force value without breaking was determined for two edges of rat skin sutured with two stitches using a two-layer thread and was found to be $0.5 \pm 0.1 \mathrm{~N}$ (Figure 4c). In addition, we checked whether the thread showed adhesion to the tissue when in a swollen state (hydrogel). To achieve this, threads were arranged between the two pieces of rat skin and after applying slight pressure a $20 \mathrm{~mm}$ plate, it was lifted at a speed of $5 \mathrm{~mm} / \mathrm{min}$, and the adherence between the fixed tissue parts was observed (Figure 4d). Therefore, it was concluded that the AlBA/TA hydrogel thread could fulfill a useful role in tissue suturing. For further discussion, the polysaccharide thread-based suturing can face challenges in sealing a variety of tissues, ranging from extremely soft liver tissues to highly elastic cardiac/vascular tissues against uncontrolled hemorrhage. To completely replace existing threads composed of biodegradable poly (lactic-co-glycolic acid), the mechanical toughness of the polysaccharide hydrogel fibers should be enhanced. 


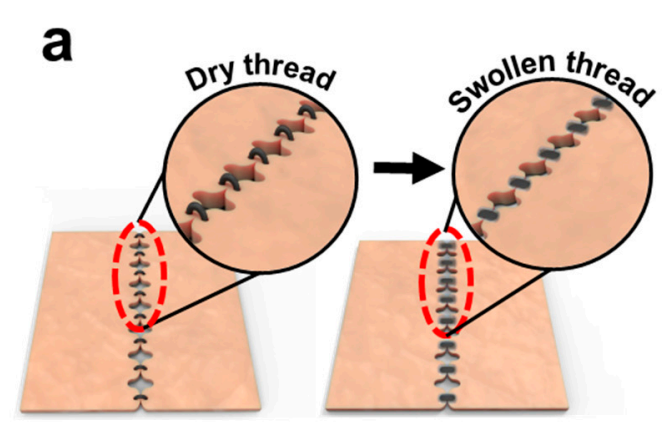

b Dry thread

d

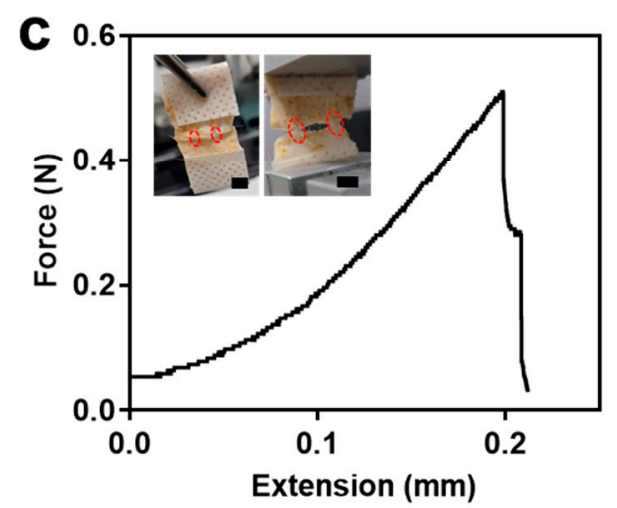

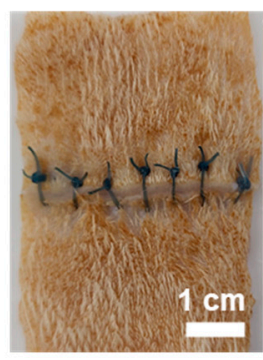

Swollen thread
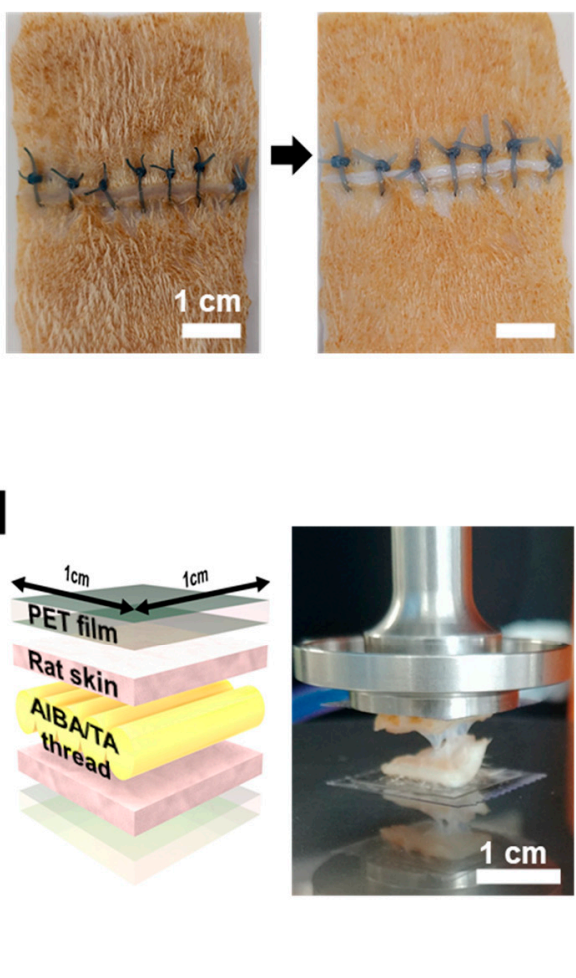

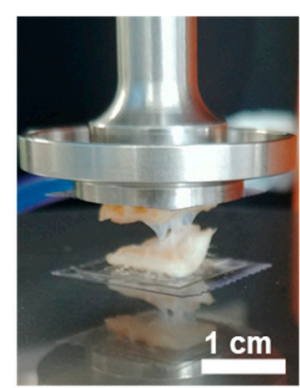

Figure 4. (a) Schematic description for ex vivo suturing test using the AlBA/TA hydrogel threads. Dried (left) and swollen suturing after exposure to PBS (pH 7.4) (right). (b) The seven-stitched suturing of rat dorsal skins by the adhesive threads. (c) Force $(\mathrm{N})$-extension $(\mathrm{mm})$ curve of the sutured skins with two stitches using two-layered AlBA/TA threads (scale bar $=0.5 \mathrm{~cm}$ ). (d) Schematic illustration for evaluating tissue-adhesiveness of the AlBA/TA threads (left) and photograph showing the adhesiveness of the swollen threads to subcutaneous tissues (right).

\section{Conclusions}

In this study, we proved the properties and functionality of hydrogel threads prepared by mixing AlBA and TA. Merely by mixing, a viscoelastic hydrogel made of AlBA reversibly crosslinked with TA was successfully used to seal tissue after drying. Before drying, the hydrogel could be transformed into various shapes, suggesting its potential for use as a 3D platform in biomedical applications. In addition, it was possible to stably fix the tissue even when the threads were swollen after suturing, which proved their adherence to the tissue in the hydrogel state. Generally, it is difficult to transform any hydrogels into thread-like shapes. However, we have successfully demonstrated that our strategy could be applied to tissue closure by forming a viscoelastic hydrogel that can be converted into a suture material.

Author Contributions: Conceptualization, M.S.; methodology, M.S. and J.H.C.; software, J.H.C.; validation, J.H.C.; formal analysis, M.S. and J.H.C.; investigation, J.H.C.; resources, M.S. and J.H.C.; data curation, M.S. and J.H.C.; writing—original draft preparation, J.H.C.; writing—review and editing, M.S. and D.S.; visualization, J.H.C.; supervision, M.S.; project administration, M.S.; funding acquisition, M.S. and D.S. All authors have read and agreed to the published version of the manuscript.

Funding: This research was funded by the National Research Foundation of Korea (NRF) grant funded by the Korean government (MSIT) (No. 2020R1C1C1003903 and No. 2020R1C1C1005567) and the Institute of Information \& Communications Technology Planning \& Evaluation (IITP) grant funded by the Korean government (MSIT) (No. 2020-0-00261). This research was also supported by an ICT Creative Consilience program (No. IITP-2020-0-01821) supervised by the IITP (Institute for Information \& Communications Technology Planning \& Evaluation), a SMC-SKKU Future Convergence Research Program grant, and a Korea Medical Device Development Fund grant funded 
by the Korean government (the Ministry of Science and ICT, the Ministry of Trade, Industry and Energy, the Ministry of Health \& Welfare, and the Ministry of Food and Drug Safety) (No. 202012D28).

Institutional Review Board Statement: Not applicable.

Informed Consent Statement: Not applicable.

Data Availability Statement: The data presented in this study are available in article.

Conflicts of Interest: The authors declare no conflict of interest.

\section{References}

1. Kim, K.; Shin, M.; Koh, M.Y.; Ryu, J.H.; Lee, M.S.; Hong, S.; Lee, H. TAPE: A medical adhesive inspired by a ubiquitous compound in plants. Adv. Funct. Mater. 2015, 25, 2402-2410. [CrossRef]

2. Grabowski, N.; Hillaireau, H.; Vergnaud, J.; Santiago, L.A.; Kerdine-Romer, S.; Pallardy, M.; Tsapis, N.; Fattal, E. Toxicity of surface-modified PLGA nanoparticles toward lung alveolar epithelial cells. Int. J. Pharm. 2013, 454, 686-694. [CrossRef] [PubMed]

3. Chang, H.; Luo, J.; Gulgunje, P.V.; Kumar, S. Structural and functional fibers. Annu. Rev. Mater. Res. 2017, 47, 331-359. [CrossRef]

4. Nary Filho, H.; Matsumoto, M.A.; Batista, A.C.; Lopes, L.C.; de Góes, F.C.; Consolaro, A. Comparative study of tissue response to polyglecaprone 25, polyglactin 910 and polytetrafluorethylene suture materials in rats. Braz. Dent. J. 2002, 13, 86-91. [CrossRef] [PubMed]

5. Varma, S.R.; Jaber, M.; Aboufanas, S.; Thomas, S.; Al Hujailan, R.G.; Al Qaoud, S.K. Evaluating tensile strengths of absorbable suture materials in herbal solutions: An In vitro study. J. Int. Oral Health 2019, 11, 148. [CrossRef]

6. Meng, H.; Li, Y.; Faust, M.; Konst, S.; Lee, B.P. Hydrogen peroxide generation and biocompatibility of hydrogel-bound mussel adhesive moiety. Acta Biomater. 2015, 17, 160-169. [CrossRef]

7. Jing, X.; Mi, H.Y.; Lin, Y.J.; Enriquez, E.; Peng, X.F.; Turng, L.S. Highly stretchable and biocompatible strain sensors based on mussel-inspired super-adhesive self-healing hydrogels for human motion monitoring. ACS Appl. Mater. Interfaces 2018, 10, 20897-20909. [CrossRef]

8. Zhang, Z.; Ni, J.; Chen, L.; Yu, L.; Xu, J.; Ding, J. Biodegradable and thermoreversible PCLA-PEG-PCLA hydrogel as a barrier for prevention of post-operative adhesion. Biomaterials 2011, 32, 4725-4736. [CrossRef]

9. Li, L.; Wang, N.; Jin, X.; Deng, R.; Nie, S.; Sun, L.; Wu, Q.; Wei, Y.; Gong, C. Biodegradable and injectable in situ cross-linking chitosan-hyaluronic acid based hydrogels for postoperative adhesion prevention. Biomaterials 2014, 35, 3903-3917. [CrossRef]

10. Jung, H.; Kim, M.K.; Lee, J.Y.; Choi, S.W.; Kim, J. Adhesive hydrogel patch with enhanced strength and adhesiveness to skin for transdermal drug delivery. Adv. Funct. Mater. 2020, 30, 2004407. [CrossRef]

11. Abebe, M.W.; Appiah-Ntiamoah, R.; Kim, H. Gallic acid modified alginate self-adhesive hydrogel for strain responsive transdermal delivery. Int. J. Biol. Macromol. 2020, 163, 147-155. [CrossRef]

12. Do, M.; Im, B.G.; Park, J.P.; Jang, J.H.; Lee, H. Functional polysaccharide sutures prepared by wet fusion of interfacial polyelectrolyte complexation fibers. Adv. Funct. Mater. 2017, 27, 1702017. [CrossRef]

13. Mirabedini, A.; Foroughi, J.; Romeo, T.; Wallace, G.G. Development and characterization of novel hybrid hydrogel fibers. Macromol. Mater. Eng. 2015, 300, 1217-1225. [CrossRef]

14. Naficy, S.; Le, T.Y.L.; Oveissi, F.; Lee, A.; Hung, J.C.; Wise, S.G.; Winlaw, D.S.; Dehghani, F. Highly porous, biocompatible tough hydrogels, processable via gel fiber spinning and 3D gel printing. Adv. Mater. Interfaces 2020, 7, 1901770. [CrossRef]

15. Wu, Y.; Shah, D.U.; Liu, C.; Yu, Z.; Liu, J.; Ren, X.; Rowland, M.J.; Abell, C.; Ramage, M.H.; Scherman, O.A. Bioinspired supramolecular fibers drawn from a multiphase self-assembled hydrogel. Proc. Natl. Acad. Sci. USA 2017, 114, 8163-8168. [CrossRef]

16. Huang, Y.; Wang, Y.; Sun, L.; Agrawal, R.; Zhang, M. Sundew adhesive: A naturally occurring hydrogel. J. R. Soc. Interface J. R. Soc. Interface 2015, 12, 20150226. [CrossRef] [PubMed]

17. Krogsgaard, M.; Nue, V.; Birkedal, H. Mussel-inspired materials: Self-healing through coordination chemistry. Chem.-Eur. J. 2016, 22, 844-857. [CrossRef] [PubMed]

18. Tønnesen, H.H.; Karlsen, J. Alginate in drug delivery systems. Drug Dev. Ind. Pharm. 2002, 28, 621-630. [CrossRef]

19. Hong, S.H.; Kim, S.; Park, J.P.; Shin, M.; Kim, K.; Ryu, J.H.; Lee, H. Dynamic bonds between boronic acid and alginate: Hydrogels with stretchable, self-healing, stimuli-responsive, remoldable, and adhesive properties. Biomacromolecules 2018, 19, $2053-2061$. [CrossRef] [PubMed]

20. Blandino, A.; Macias, M.; Cantero, D. Formation of calcium alginate gel capsules: Influence of sodium alginate and CaCl2 concentration on gelation kinetics. J. Biosci. Bioeng. 1999, 88, 686-689. [CrossRef]

21. Hong, S.H.; Shin, M.; Park, E.; Ryu, J.H.; Burdick, J.A.; Lee, H. Alginate-boronic acid: pH-triggered bioinspired glue for hydrogel assembly. Adv. Funct. Mater. 2020, 30, 1908497. [CrossRef]

22. Ovesen, L.; Bendtsen, F.; Tage-Jensen, U.; Pedersen, N.T.; Gram, B.R.; Rune, S.J. Intraluminal pH in the stomach, duodenum, and proximal jejunum in normal subjects and patients with exocrine pancreatic insufficiency. Gastroenterology 1986, 90, 958-962. [CrossRef] 
23. Ilbekwe, V.C.; Fadda, H.M.; McConnell, E.L.; Khela, M.K.; Evans, D.F.; Basit, A.W. Interplay between intestinal pH, transit time and feed status on the in vivo performance of $\mathrm{pH}$ responsive ileo-colonic release systems. Pharm. Res. 2008, 25, 1828-1835. [CrossRef]

24. Yeo, J.; Lee, J.; Yoon, S.; Kim, W.J. Tannic acid-based nanogel as an efficient anti-inflammatory agent. Biomater. Sci. 2020, 8, 1148-1159. [CrossRef]

25. Akiyama, H.; Fujii, K.; Yamasaki, O.; Oono, T.; Iwatsuki, K. Antibacterial action of several tannic acids against Staphylococcus aureus. J. Antimicrob. Chemother. 2001, 48, 487-491. [CrossRef] [PubMed]

26. Owens, C.D.; Stoessel, K. Surgical site infections: Epidemiology, microbiology and prevention. J. Hosp. Infect. 2008, 70, 3-10. [CrossRef]

27. Edmiston, C.E., Jr.; Krepel, C.J.; Marks, R.M.; Rossi, P.J.; Sanger, J.; Goldblatt, M.; Graham, M.B.; Rothenburger, S.; Collier, J.; Seabrook, G.R. Microbiology of explanted suture segments from infected and noninfected surgical patients. J. Clin. Microbiol. 2013, 51, 417-421. [CrossRef] [PubMed] 